\title{
Schamanentrachten in Sibirien
}

\author{
Formen und Funktionen, Alter und Herkunft
}

BY Bo LöNNQvisT

1

In der 1980 herausgegebenen, bearbeiteten und erweiterten Auflage seiner Arbeit ,,Schamanen und Medizinmänner. Magie und Mystik früher Kulturen“ behandelt der Museumsforscher Andreas Lommel im Kapitel ,,Kunst und Schamanismus" auch ,Schamanenkostüme und Masken" (Lommel 1980, 162-175). Das Kapitel stellt eigentlich eine Zusammenfassung früherer Forschung dar. Lommel betont drei Faktoren: 1) das Vorkommen einer ,,wirklichen" Schamanentracht einzig in Sibirien, 2) die Funktion der Tracht, die nach Lommels Ansicht in erster Linie psychologisch-künstlerischer Art ist und 3) die Symbolik der Tracht. Lommel betont den Charakter der Tracht als Tierverkleidung und weist darauf hin, daß,,die älteste Darstellung eines Schamanen" - nämlich die Höhlenmalerei von Trois Frères - ,einen Schamanen in Tierverkleidung zeigt" (Lommel 1980, 165). Weiterhin verweist er auf den Zusammenhang zwischen der Dekorierung der Tracht mit Tierfiguren und der schamanistischen Vorstellung von Hilfsgeistern in Tiergestalt. Diese weiter existierende oder wiederbelebte Skelettmagie vergleicht er mit Felsenmalereien und lappischen Trommeln der gleichen Art. Laut Lommel deutet die Skelettmagie auf die Auffassung der Jägervölker von der Wiederauferstehung des getöteten Tieres hin. Die figürlichen Gehänge an der Tracht bilden folglich Symbole für die Hilfsgeister in Tiergestalt, und deren Eigenschaften können im Zustand der Trance auf den Schamanen übergehen. Unter Hinweis auf eiserne Gehänge an den jakutischen, tungusischen, burjatischen und jenissei-ostjakischen Trachten stellt Lommel fest, daß die ,,alten, vollständigen Trachten " - seiner Meinung nach ähneln sich Schamanentrachten im allgemeinen - ein Tier darstellen und daß Haupttypen der Tierverkleidungen Vogel, Hirsch und Bär sind. Lommel stützt sich hier auf die Theorie von Uno Harva (Harva 1922; Harva 1938), der wiederum auf Gedanken aufbaute, die schon 1925 von Nioradze und 1902 von Troščanskij vorgebracht wurden. Ebenso stützt sich Lommel auf die Deutung der Funktion der Tracht durch A. Friedrich (Friedrich 1943-44). Laut eigenen Berichten sibirischer Schamanen sowie 
ausgehend von den ekstatischen Tänzen während der Séance kann die Tracht gedeutet werden als ein Mittel, mit dessen Hilfe sich der Schamane in ein Tier verwandelt; ein atavistisches und somit altes Merkmal im Schamanismus. Die Wiedergeburt des Schamanen wiederum - den Berichten zufolge geschieht sie aus eigenem Skelett und Fleisch sowie aus dem Skelett und Fleisch der Stammesangehörigen - zeigt dessen menschliche Natur; auch dies kann durch die Tracht veranschaulicht werden. Eine ähnliche zusammenfassende Deutung gibt Eliade mit seiner Feststellung, daß die Tracht einerseits ein symbolisches System bildet, andererseits - als Werkzeug - verschiedene geistige Kräfte besitzt. Auch Eliade neigt dazu, die sibirischen Schamanentrachten in eine gewisse Entwicklungsreihe einzuordnen. Den vollständigsten und am besten erhaltenen Typ soll es danach unter den Altai-Stämmen geben, während die tungusischen Trachten Merkmale von den jakutischen und burjatischen Trachten erhalten haben sollen. Von den Kupferspiegeln an den Trachten der südlichen Tungusen nimmt man an, daß sie sino-mandschurischen Ursprungs seien (Eliade 1970, 145-180).

\section{2}

Innerhalb der Religionsforschung sind die sibirischen Schamanentrachten vor allem als Dokument bei der Bestimmung des Wesens, des Alters und des Ursprungs des Schamanismus interessant geworden. Aus dem relativ heterogenen Material hat man einen Idealtyp einer Tracht geschaffen, obwohl es bei der rituellen schamanistischen Bekleidung in Sibirien große Unterschiede gibt und obwohl rituelle Trachten in Verbindung mit ekstatischer Religionsausübung auch in anderen Kulturen vorkommen. Ebenso berücksichtigt diese Betrachtungsweise nicht die lange und unterschiedliche Bevölkerungs- und Siedlungsgeschichte der sibirischen Völker. Auch sollte das Verhältnis der Tracht zu anderen Attributen - vor allem der Trommel - analysiert werden, ebenso die Frage, warum Trachten dieses Typs nur in Sibirien vorkommen, obwohl ähnliche Merkmale innerhalb des Schamanismus als Religionsform auch in anderen Gebieten - u. a. in Lappland - verbreitet sind. Ferner scheint die Deutung der zur Tracht gehörenden Figuren nicht so klar zu sein, wie man bisher annahm (vgl. Ivanov 1978; vgl. Ivanov 1963; vgl. Ivanov 1970; vgl. Ivanov 1979).

Hans Findeisen, Vilmos Diószegi und Helmut Hoffman haben die Schamanentrachten auch unter historischen Aspekten untersucht. Findeisen bringt Harvas Theorie mit ethnischen Fakten in Verbindung und ordnet daher die Trachten vom Typ Vogel den Gruppen mit Turksprachen zu (Altai, Jakuten) und die vom Typ gehörntes Reh sowie Rentier den Samoje- 
den, Tungusen und Burjaten. Nach Findeisen ist die Vogelmaske die älteste Trachtenform. Diese Aussage wird sowohl durch die Funktion der Tracht als Hilfsmittel während der Reise als auch durch die totemistischen Berichte über den Adler als Gebärerin des Schamanen unterstützt. Die prähistorischen Abbildungen zeugen jedoch von anderen Tierverkleidungen, und zwar von Fellen, Geweihen und Masken. Trotzdem können die Jägerkulturen in Sibirien mit den prähistorischen Jägerkulturen in Verbindung gebracht werden, und die Schamanentrachten können mit der Geschichte der Tierverkleidung verglichen werden, welche während des jüngeren Paläolithikums (Magdalenien ca. 8500 v. Chr.) begann (Findeisen 1957, 80-85).

In seiner Untersuchung des Schamanismus in der Mongolei betont Diószegi den unterschiedlichen etnischen Ursprung der Stämme sowie deren sprachliche Differenzierung. Eine zentrale Frage ist laut Diószegi jedoch die, ob die Formen des Schamanismus als konservativ genug angesehen werden können, um ethnogenetische Probleme erhellen zu können. Die Burjaten, Tungusen und die Turkstämme leben ja über ganz Mittelasien und Sibirien verstreut. Außer auf interethnische Akulturationsprobleme weist Diószegi auch auf die interreligiöse Problematik hin, die das Verhältnis des ursprünglichen Schamanismus zum vordringenden Buddhismus betrifft. Auch Ulla Johansen und Eliade haben ähnliche Gesichtspunkte vorgebracht sowie mögliche kulturelle Einflüsse aus Tibet, China und der Mongolei angedeutet (Diószegi 1961, Johansen 1954, Eliade 1970).

In seiner Arbeit ,Symbolik der tibetischen Religionen und des Schamanismus" schließt sich Helmut Hoffman Harvas Auffassung darüber an, daß die Schamanentracht in späterer Zeit degenerierte. Er nimmt an, daß die Tracht sowie die Masken in einem weiten arktischen Gebiet von Nordsibirien bis nach Lappland vorkamen; dort hat sich die Trommel bis heute erhalten. Bei seiner Kritik der Diskussion Harvas und Findeisens über die Tiersymbolik widmet Hoffmann den Metallgehängen zentrale Aufmerksamkeit; er behauptet, daß diese relativ späte Requisiten seien, hinzugefügt, , als die alte Bedeutung des Schamanengewandes nicht mehr vollkommen bekannt war" (Hoffmann 1967, 131-140).

Die früheren Deutungen der Schamanentracht repräsentieren damit zwei verschiedene Betrachtungsweisen. Einerseits hat man die Funktion der Tracht als Inkarnation des Schutzgeistes - des Tieres - hervorgehoben (Troščanskij, Nioradze, Harva, Lommel), andererseits war man der Meinung, daß die Trommel der Vorgänger der Tracht war und man nahm an, daß die Tracht nach und nach ihre reich ausgearbeitete Form erhalten hat 
(Stadling 1912, nach Pekarskij und Vasiljev 1910). Die eine Betrachtungsweise betont die Funktion der Tracht, die andere historisch-geographische Faktoren.

Des weiteren muß folgendes beachtet werden:

1. Bei Samojeden, Tschuktschen und gewissen anderen paläo-asiatischen Völkern (Nanaitsi) gibt es keine reich dekorierten, ausgesprochen rituellen Trachten in dem Sinne wie z. B. bei den eigentlichen Tungusen, den Altai-Völkern, den südlichen Tungusen und den Burjaten.

2. Die Festlegung dessen, was eine ,,eigentliche" Schamanentracht ist, wird auch durch die Tatsache erschwert, daß ein Schamane seine Tracht sein ganzes Leben lang vervollständigte. Während der Zeit, in der die Schamanentracht außer Gebrauch kam, also im 20. Jahrhundert, haben die Museen auch sehr einfache und fragmentarische Schamanentrachten erworben.

Der überwiegende Teil der in Museen aufbewahrten Schamanentrachten aus Sibirien wurde zwischen ca. 1860 und 1920 gesammelt. Ich hatte Gelegenheit, in Museen in Europa und Sibirien ca. 130 erhaltene Trachten sowie Trachtenteile (Kopfbedeckungen, Brustlatze, Handschuhe, Stiefel) zu besichtigen. Darüber hinaus sind ca. 50 Trachten wissenschaftlich publiziert worden. Ein Vergleich zwischen dem ältesten Bildmaterial (z.B. Witzen 1705, Georgi 1772-74, Pallas 1776) und dem musealen Material zeigt, daß die Tracht vom Ende des 18. bis Ende des 19. Jahrhunderts keine Veränderungen erfahren hat. Dagegen sind die Veränderungen im 20. Jahrhundert beträchtlich; sie bestehen hauptsächlich in einer Vereinfachung der Gestaltung. Weiter kann festgehalten werden:

1. Nicht alle Schamanen hatten besondere rituelle Trachten; bei einem Teil beschränkt sich das rituelle Merkmal auf die Kopfbedeckung. Dagegen ist die Trommel obligatorisch.

2. Die im ältesten musealen Material vorkommenden echten Tierteile (Krallen, Flügel, Pfoten) waren möglicherweise früher mehr verbreitet; darauf deuten auch die Bilder bei Witzen und Georgi hin, soweit sie zuverlässig sind.

Im folgenden sollen die Schamanentrachten in Sibirien nicht unter dem Gesichtspunkt der Religionsforschung untersucht werden, sondern unter dem Gesichtspunkt der Trachtenethnologie. Bei Beachtung der oben referierten Forschung ist die zentrale Frage hier nicht die nach dem Beweiswert der Tracht bezüglich des Wesens, des Alters und des Ursprungs des 
Schamanismus, sondern die umgekehrte Frage, nämlich die nach dem Bedarf des Schamanismus, der rituellen Ekstasetechnik, an einer rituellen Tracht überhaupt.

Welche Schlüsse kann man aufgrund des Alters, der Form und der Verbreitung des erhaltenen Trachtenmaterials ziehen? In welchem Umfang kommen rituelle Trachten in Verbindung mit dem Schamanismus in Sibirien vor, und kann man überhaupt von einem bestimmten Typ von Schamanentracht sprechen?

Welche Funktion hat die Tracht bei der Séance und auf welche Weise wird die Tracht durch die übrige Ausstattung ergänzt, d. h. in welchem Maß bildet die Tracht eine notwendige Ergänzung zu den übrigen Requisiten, insbesondere zum Gebrauch der Trommel?

Im Blickpunkt der Forschung steht also die Funktion der Tracht als Mittel der Transformation sowohl für den Schamanen als auch zwischen Schamane und Zuschauer, eine Funktion, die die Trommel oder ein anderes Werkzeug nicht zu haben scheint. Gemeinsam für Trommel und Tracht ist jedoch, daß beide, wenn auch in verschiedener Form, das Weltbild des Schamanen, den Kosmos, veranschaulichen. Eine dekorierte Trommel und eine Tracht mit figursymbolischer Verzierung schließen sich also zu einem gewissen Grad gegenseitig aus, sie bilden alternative Ekstasewerkzeuge. Aus visueller Perspektive dürfte die Trommel jedoch eine direktere Beziehung zum Schamanen haben, während die Tracht sich ja visuell in hohem Maße an die Zuschauer wendet. Als persönliches Mittel zur Verwandlung dagegen ist die Tracht zentralere, während die Trommel wiederum die Funktion eines Beförderungs-mittels für den Schamanen haben kann. Betreffend der Geräuscheffekte ist die Trommel dominierend, aber auch die mit Metallgehängen reich dekorierten Trachten haben die Gleiche Funktion.

Wenn man die Tracht als ein Kodesystem ansieht, das die zweifache Funktion hat, sowohl die innere Existenz des Individuums/des Schamanen zu befördern als auch mit seiner Stellung gegenüber der sozialen Umwelt übereinzustimmen (vgl. Enninger 1983), wird die Frage nach der Notwendigkeit der Tracht noch zentraler. Im Blickpunkt steht dann die Rolle des Schamanen und des Schamanismus in der Gesellschaft überhaupt.

Vom trachtengeographischen Aspekt aus kann festgehalten werden, daß der überwiegende Teil des erhaltenen Trachtenmaterials aus dem südlichen Sibirien stammt, aus einer Zone, die vom Gebiet Altai/Baikal bis zum Amur reicht. Auch das museale Material wird im wesentlichen von zwei Völkergruppen repräsentiert: 1) Tungusen (Krasnojarsk, Zabajkal, Irkutsk) und 2) verschiedene ethnische Gruppen im Gebiet des Altai, der Sajan-Berge und in der nördlichen Mongolei. Darauf folgt das Material von den samojedischen Stämmen, den Jakuten und den südlichen Tungusen. Die meisten 
Variationen bezüglich der Ausgestaltung und des Materials der Trachten findet man bei den zwei erstgenannten Gruppen. Ich habe das Trachtenmaterial in vier Gruppen eingeteilt, welche im wesentlichen der Einteilung von E. Prokofyeva aufgrund des Materials im Muzej Antropologij i Etnografij zu entsprechen scheint (Prokofyeva 1971; vgl. Lönnqvist 1976).

1. Zur ersten Gruppe können die Trachten der eigentlichen Tungusen gerechnet werden (Oberlauf des Jenissej, Angara);

2. zur zweiten Gruppe die Trachten der kleinen ethnischen Gruppen im Gebiet des Altai (Sojoten, Karagassen etc.);

3. zur dritten Gruppe die Trachten der südlichen Tungusen und der Burjaten in der nördlichen Mongolei, in der nördlichen Mandschurei sowie die der Stämme am Unterlauf des Amur;

4. zur vierten Gruppe die Trachten der Samojeden zwischen Ob und Jenissej, der nördlichen Tungusen, der Dolganen, Jakuten, Jukagiren, Tschuktschen usw.

Eine derartige auf der Form und der geographischen Verbreitung der Trachten beruhende kartographische Aufnahme ergibt folgendes Bild:

1. Je weiter man sich vom Gebiet Altai/Baikal/Amur entfernt in Richtung der Samojeden im Nordwesten und der Paläo-Asiaten im nordöstlichen Sibirien sowie am Unterlauf des Amur, umso weniger ausgeprägt ist die Verwendung einer speziellen rituellen Tracht in Verbindung mit dem Schamanismus.

2. Viele Details der Schamanentracht - wie hängende Bänder, Schellen, Stickereien aus Perlen oder Rentierhaaren, verzierte Stiefel, Handschuhe, Kopfbedeckungen - sind bei tungusischen, samojedischen, jakutischen Trachten sowie denen der Turkvölker allgemein verbreitet und haben keine ausschließlich rituelle Funktion.

3. Als zentrale symbolische Elemente der Schamanentracht bleiben damit noch die metallischen oder aufgemalten oder aufgestickten Tierfiguren auf dem Kaftan oder der Kopfbedeckung übrig.

4. Eine ausgesprochen andersartige und in dieser Hinsicht „eigentliche“ rituelle Tracht in Verbindung mit dem Schamanismus dürfte in erster Linie typisch sein für die Tungusen und die mit ihnen in Berührung stehenden Jakuten und Burjaten sowie für die Altai-Völker.

\section{5}

Eine ausschlaggebende Frage bei der Analyse der Ausgestaltung der Schamanentracht nach historischen, ökologischen und religiös-sozialen Gesich- 
tspunkten betrifft das Verhältnis der Tracht zur Trommel als ein Werkzeug, das sowohl das Weltbild veranschaulicht als auch die verbale und musikalische Tradition während der Ekstase veranschaulicht. Diese Sichtweise vereinigt sowohl eine mehr zeitlose funktionalistische Perspektive als auch eine kulturhistorische Perspektive.

Es ist wichtig festzustellen, bei welchen Gruppen und in welchen Gebieten das Transformationsthema der Séance eine spezielle, rituelle Tracht entwickelt hat und die Bilderwelt der Trommel auf die der Tracht übergegangen ist. Könnte es möglicherweise so sein, daß ein primärer, nomadistischer Jagdritus und die Ahnenverehrung keine spezielle Tracht voraussetzten, sondern die Trommel als wichtigstes Ekstasewerkzeug und Symbol des Weltbilds fungierte? Verschiedene Forscher haben ja auch die Funktion der Trommel als Reittier für die Reise des Schamanen betont (Kamel, Elch, Pferd) (vgl. Potapov 1978). Folglich müßte dann die eigentliche Schamanentracht in Sibirien mehr mit dramatischen religiösen Techniken zusammenhängen (öffentlicher Auftritt) sowie mit einer Situation, in der die Rolle des Schamanen Merkmale eines Berufs besitzt (vgl. Weinstein 1963). Die Schamanentracht könnte dann als ein nördliches Parallelphänomen zu den rituellen Trachten der Glaubensformen Mittelasiens, z. B. des Lamaismus und der damit verschmolzenen Bon-Religion, angesehen werden sowie zu den bei den Cham-Zeremonien in der Inneren Mongolei verwandten Trachten. Trotzdem ist der rituelle Symbolwert der sibirischen Schamanentrachten ein anderer, obwohl ihre Funktion, ihren Träger, darin besteht, zu einem nicht menschlichen Wesen zu transformieren. Die Trommel hat in diesem Zusammenhang nur eine begleitende Funktion; der Blick ist auf die Tracht gerichtet. Von diesem Gesichtspunkt aus gesehen könnte man daher bei den in Verbindung mit der schamanistischen Ekstase in Sibirien vorkommenden Requisiten zwei Traditionsschichten unterscheiden, einerseits ein archaisches Stadium, andererseits ein Stadium, in dem eine berufsmäßige Ekstasetechnik eine ihr entsprechende Ausrüstung hervorgebracht hat. Die rituelle Tracht würde also nach dieser Betrachtungsweise das letztgenannte Stadium vertreten, d. h. sie würde ein Produkt einer religiösen Hochkultur darstellen und die Schamanentracht wäre demnach ein relativ spätes Phänomen bei den sibirischen Völkern. Gemäß einer solchen Betrachtungsweise hat sich eine speziell rituelle Tracht in Verbindung mit dem Schamanismus z. B. der Lappen niemals entwickelt. Vom obengenannten Gesichtspunkt aus gesehen erscheint dann auch ein Vergleich zwischen den sibirischen Schamanentrachten und den Tierverkleidungen in den prähistorischen Höhlen- und Felszeichnungen als ziemlich spekulativ. 


\section{Literaturverzeichnis}

Diószegi, V. 1961. A mongóliai samanizmus kérdései (Die Fragen des mongolischen Schamanismus). Ethnographia 72, 4.

Eliade, M. 1970. Shamanism. Princeton.

Enninger, W. 1983. Kodewandel in der Kleidung. Zeitschrift für Semiotik 5, 1-2. Findeisen, H. 1957. Schamanentum. Stuttgart.

Friedrich, A. 1943-44. Knochen und Skelett in der Vorstellungswelt Nordasiens. Wiener Beiträge zur Kulturgeschichte und Linguistik 5.

[Harva], U. 1922. The shaman costume and its significance. By U. Holmberg. (Turun suomalaisen yliopiston julkaisuja B, 1, 2.) Turku.

- 1938. Die religiösen Vorstellungen der altaischen Völker. (FF Communications 125.) Helsinki.

Hoffman, H. 1967. Symbolik der tibetischen Religionen und des Schamanismus. (Symbolik der Religionen 12.) Stuttgart.

Ivanov, S. V. 1963. Ornament narodov Sibiri. (Trudy instituta etnografii. Novaja serija 81.) Moskva.

- 1970. Skulptura narodov severe Sibiri. Leningrad.

- 1978. Some aspects of the study of Siberian shamanism. Shamanism in Siberia. Ed. by V. Diószegi and M. Hoppál. Budapest.

- 1979. Skulptura altajtsev, hakasov i sibirskih tatar. Leningrad.

Johansen, U. 1954. Die Ornamentik der Jakuten. (Wegweiser zur Völkerkunde 3. Museum für Völkerkunde und Vorgeschichte.) Hamburg.

Lommel, A. 1980. Schamanen und Medizinmänner. München. Vgl. da angeführte Literatur.

Lönnqvist, B. 1976. Problems concerning the Siberian shaman costume. Etnologia Fennica 1-2.

Nioradze, G. 1925. Der Schamanismus bei den sibirischen Völkern. Stuttgart.

Potapov, L. P. 1978. The shaman drum as a source of ethnographical history. Shamanism in Siberia. Ed. by V. Diószegi and M. Hoppál. Budapest.

Prokofyeva, E. D. 1971. Samanskie kostjomy narodov Sibiri. (Sbornik Muzeja Antropologii i Etnografii 27.) Leningrad.

Stadling, J. 1912. Shamanismen i norra Asien. (Populära etnologiska skrifter 7.) Stockholm. Siehe da angeführte Literatur.

Weinstein, S. I. 1963. Die Schamanentrommel der Tuwa und die Zeremonie ihrer „,Belebung“. Glaubenswelt und Folklore der sibirischen Völker. Hrsg. von V. Diószegi. Budapest. 